\title{
A Digital Pen with a Trajectory Recognition Algorithm
}

\author{
Nandini ${ }^{1}$, G. Bhaskar Phani Ram ${ }^{2}$ (Asst. professor) \\ ${ }^{1,2}$ (Department of ECE, Vardhaman College of Engineering (Autonomous), JNTU Hyderabad, India)
}

\begin{abstract}
Now a days, the development of miniaturization technologies in electronic circuits and components has seriously decreased the dimension and weight of consumer electronic products, those are smart phones and handheld computers, and thus prepared them more handy and convenient. This paper contains an accelerometer-based digital pen for handwritten digit and gesture trajectory recognition applications. The digital pen consists of a triaxial accelerometer, a microcontroller, and an Zigbee wireless transmission module for sensing and collecting accelerations of handwriting and gesture trajectories. with this project we can do human computer interaction.

Users can utilize this pen to write digits or make hand gestures, and the accelerations of hand motions calculated by the accelerometer are wirelessly transmitted to a computer for online trajectory recognition. So, by varying the position of mems (micro electro mechanical systems) we can capable to show the alphabetical characters in the PC. The acceleration signals calculated from the triaxial accelerometer are transmitted to a computer via the wireless module.
\end{abstract}

Keywords - ARM, Zigbee, Sensors module

\section{Introduction}

Most motion tracking systems are utilized for outdoor applications such as missile or vehicle tracking. Recently, motion tracking systems have been researched on various indoor applications such as manufacturing human computer interaction.

Pen based input devices embedded with mems sensors have been provided for hand gestures or hand writing. A silent application of mems sensors for general motion sensing is that they can be operated with low voltage.

The acceleration signals measured from the triaxial accelerometer are transmitted to a computer via the wireless module. The measured acceleration signals of these motions can be recognized by the trajectory recognition algorithm. A mems based digital pen, and a trajectory recognition algorithm have been developed in this paper. The digital pen consist of a triaxial accelerometer, a microcontroller and a Zigbee wireless transmission module. The measured raw signals from the sensors are transmitted to a computer via the wireless module. Users can use the digital pen to write numerals, alphabets at normal speed without any space limitation. The movement trajectories can be reconstructed by the trajectory recognition algorithm. The advantages of our proposed human computer interaction input device include the following:1)It is portable and can be used anywhere without any external reference device or writing ambit limitations, and 2)the trajectory recognition algorithm can reduce orientation and integral errors effectively and thus can reconstruct the trajectories of movements accurately.

The arrangement of this paper is as follows, First the particulars of the hardware device, the accelerometer based digital pen for handwritten, microcontroller, zigbee transmission and trajectory recognition algorithm in detail, parallelly human computer interaction application respectively.

\section{Digital Pen Components And Its Basic Operational Algorithm}

In the following subsections, we describe the hardware system design using MMA7361accelerometer, to evaluate the accelerometer's performance, LPC2148 microcontroller, the controller collects the analog acceleration signals and converts the signal to digital signal via the A/D converter. The transmitter transmits the acceleration signals wirelessly to a personal computer, and the algorithm to measure the displacement from the acceleration. 


\subsection{Block Diagram:}

\subsubsection{Pen section:}

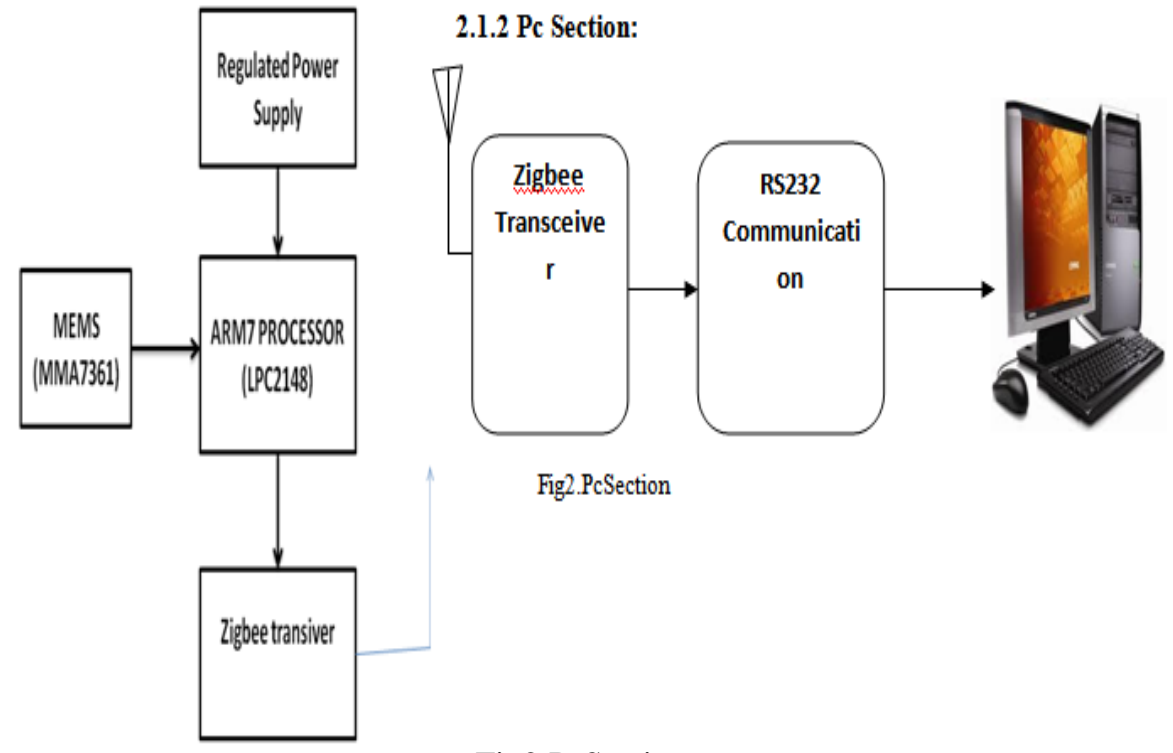

Fig2.PcSection

Fig1.Block Diagram of Pen section

\subsection{Hardware Description}

The hardware system is shown in Fig.1 and Fig.2. In the laboratory developed board, the power supply circuit built using filters, rectifiers and then voltage regulator, with an AC voltage, a steady DC voltage is obtained by rectifying the AC voltage then filtering to a DC level and finally regulating to obtain a desired, fixed Dc voltage. the accelerometer placed to measure the acceleration in three directions, and those signals are transmitted to microcontroller and converts the signals to digital form and transmitted to a computer via the wireless module by using Zigbee technology.

\subsection{Lpc2148 Microcontroller}

LPC2148 Microcontroller Architecture. The ARM7TDMI-S is a general purpose 32-bit microprocessor, with real time emulation and embedded high-speed flash memory rangig from $32 \mathrm{kB}$ to 512KB.A 128 bit wide memory interface and a unique accelerator architecture enable 32-bit code execution at the maximum clock rate. This simplicity results in a high instruction throughput and impressive real-time interrupt response from a small and cost-effective processor core.

Pipeline techniques are engaged so that every part of the processing and memory systems can operate endlessly. usually, while one instruction is being executed, its descendant is being decoded, and a third instruction is being fetched from memory. The ARM7TDMI-S processor also employ a single architectural stratagem known as Thumb, which makes it perfectly suited to high-volume applications with memory restrictions, or applications where code density is a matter.

The key idea following Thumb is that of a super-reduced instruction set. Basically, the ARM7TDMI-S processor has two instruction sets:

- The standard 32-bit ARM set.

- A 16-bit Thumb set.

The Thumb set's 16-bit instruction length allow it to move toward double the density of standard ARM code at the same time as retaining most of the ARM's performance advantage over a recognized 16-bit processor using 16-bit registers. This is probable because Thumb code operates on the same 32-bit register set as ARM code. Thumb code is capable to provide up to $65 \%$ of the code size of ARM, and $160 \%$ of the performance of an the same ARM processor connected to a 16-bit memory system 


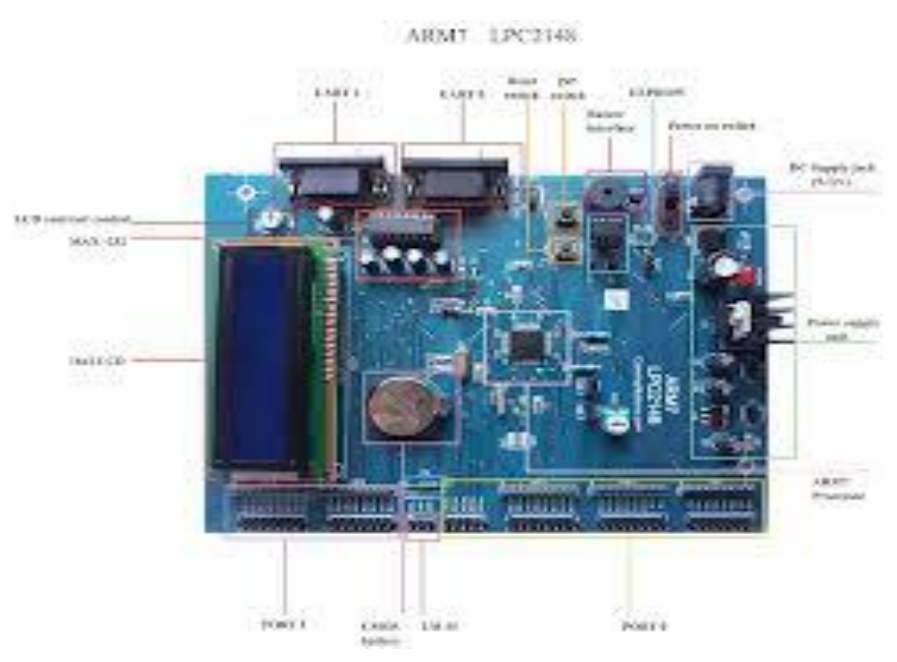

III. MEMS Technology:

Micro-Electro-Mechanical Systems (MEMS) is the combination of mechanical elements, sensors, actuators, and electronics on a common silicon substrate for the period of micro fabrication technology.MEMS is an enabling technology allowing the growth of stylish products, augmenting the computational capability of microelectronics. In the fashionable cases, the physics behind the performance of MEMS devices can be expressed by mathematical terminology. MEMS Solver mechanism by creating a mathematical model of the system and generates logical solutions to explain the presentation of the MEMS device. The user now has to enter the input parameters like length and width of the beam for example in a user friendly GUI, and the software will directly calculate the relevant results and plot graphs that fully give details of the MEMS device or part of it.

The software is divided into five modules, they are mechanics, sensing, actuation, and process and data analysis. Mechanics module be subdivided into three sub sections. The first subsection being structures where the mainly used beams and diaphragm designs are examined. The second subsection explains the vibration of these structures, these two are free and forced vibrations. The third subsection explains damping in the form of squeeze film and slide film damping. Sensing module explains sensing schemes generally used in MEMS namely piezoresistive and capacitive sensing for scheming pressure sensors and accelerometers. Actuation module examines the both widely used means of actuation namely electrostatic and thermal applied to some frequently used actuators like parallel plate, micro mirror, comb drive, bimetallic and bimorph actuators. Process module was separated into six subsections namely lithography, oxidation, diffusion, implantation, film deposition and wet etching. This covers some of the most normally used processes used in the development of MEMS devices. The data analysis module have a die calculator, unit conversion tool and lists the material properties of normally used MEMS materials

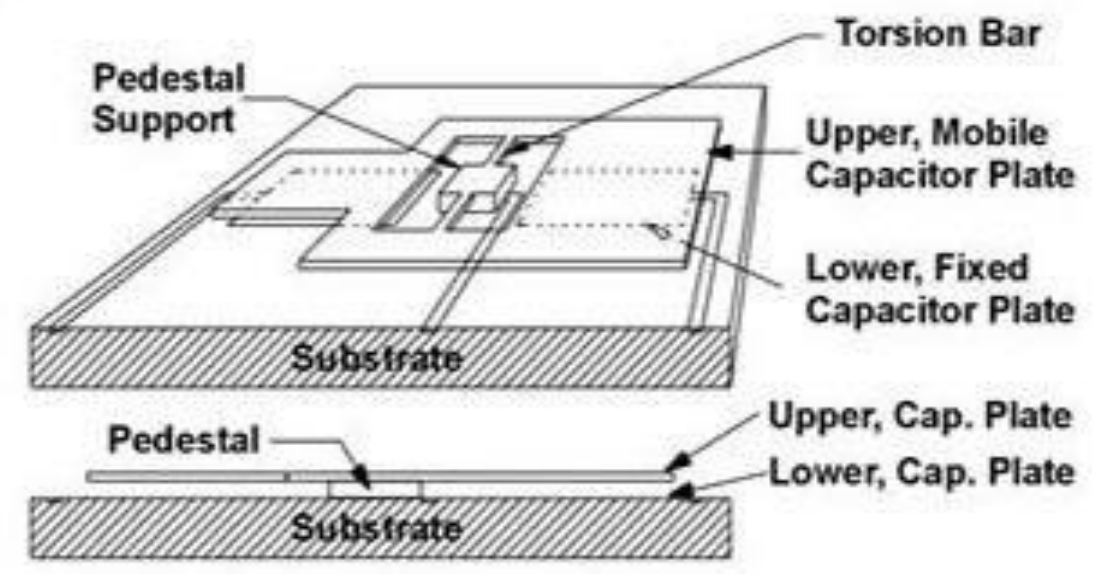

The rising demand for MEMS (micro-electromechanical systems) technology is approaching from diverse industries such as automotive, space and user electronics. MEMS promises to develop almost every product category by bringing together silicon-based microelectronics with micromachining technology, 
manufacturing the possible of the realization of complete systems-on-a-chip. KLA-Tenor offers the tools and techniques, first developed for the included circuit industry, for this rising market.

\subsection{Mems And Compliant Mems}

Micro-electro-mechanical systems (MEMS) technology has contributed to the better performance, reliability and lower-cost sensors that carry basic automobile functions within the automotive industry. MEMS technology is estimated to play an important role in the future of Research and Development of automotive industry particularly in the active safety area. MEMS sensors have the following advantages: they are deterioration-free and are long-lasting for long periods; they contain good dynamic characteristics, superior impact resistance, low power consumption, inexpensive, they are tiny in size, and simple for installation. MEMS are measured to be as a key technology with potential to meet the necessities of the Intelligent Transportation Technology (ITS). MEMS sensors used in automotive systems etc. typically consist of micro beams and inertial mass twisted by etching part of a silicon substrate, and piezo-resistors twisted as strain gauges on the beams. Applications of MEMS sensors are unlimited to airbag systems. They are also worn in vehicle motion control systems, for example in the Antilock Braking System (ABS). Crash sensors can senses and calculate crash parameters such as velocity and acceleration. Existing technologies for active safety are being personalized using MEMS sensors to enhance the show of current systems; such as airbags or belt pre-tension devices. These systems reduce the opportunity of injury and its level for the period of a crash which motivates the development of Intelligent Safety Systems (ISS). In this research two obedient MEMS designs are introduced as shown in Figures 3-1. These mechanisms work on the standard of large deflecting arcs and the beams and reach motion by the deflection of their members. Prescribed motion profiles can be obtained more simply using buckling members in compliant mechanism design. If these mechanism's people were inflexible the mechanism would have zero degree of freedom.

\section{Trajectory Recognition Algorithm}

A trajectory is the lane that a moving object follows through space as a role of time. A trajectory is a sequence $\left(f^{k}(x)\right)_{k \in \mathbb{N}}$ of values considered by the iterated application of a mapping $f$ to an element $x$ of its source.

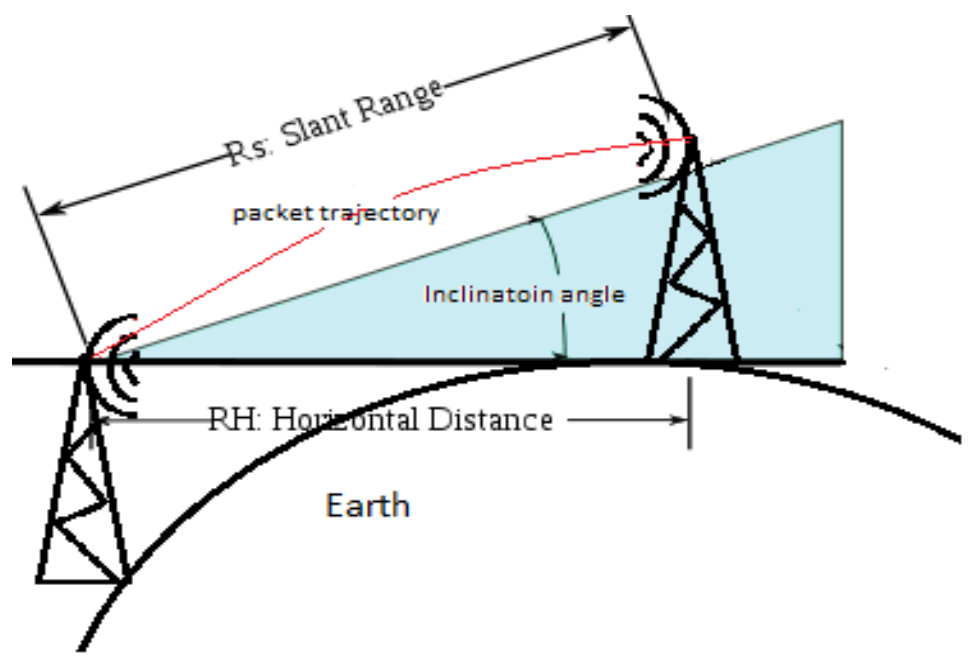

Illustration showing the trajectory of a packet at an uphill transceiver target

\subsection{Physics Of Trajectories}

Consider a particle of mass $m$, touching in a potential field $V$. Physically speaking mass represents inertia, and the field $V$ represents external forces, of a particular kind known as "conservative". That is, given $V$ at all relevant positions, there is a method to infer the associated force that would operate at that position, say from gravity. Not all forces can be explained in this way, however.

The movement of the particle is described by the second-order differential equation

$$
m \frac{\mathrm{d}^{2} \vec{x}(t)}{\mathrm{d} t^{2}}=-\nabla V(\vec{x}(t)) \text { with } \vec{x}=(x, y, z)
$$




\subsection{Uniform Gravity, No Drag Or Wind}

Assume $g$ be the acceleration, Relative to the flat terrain let the first horizontal speed will be $v_{h}=v \cos (\theta)$ and the vertical speed will be $v_{v}=v \sin (\theta)$. It will also be shown that, the range is $2 v_{h} v_{v} / g$, and the maximum altitude is $v_{v}^{2} / 2 g$; the maximum range, for a given initial speed $v$, is obtained when $v_{h}=v_{v}$, i.e. the initial angle is 45 degrees. This range is $v^{2} / g$, and the maximum altitude at the maximum range is a quarter of that.

\subsection{Derivation Of The Equation Of Motion}

Assume the motion of the projectile is being calculated from a Free fall frame which happens to be at $(\mathrm{x}, \mathrm{y})=(0,0)$ at $\mathrm{t}=0$. The equation of motion of the projectile in this frame (by the principle of equivalence) would be $y=x \tan (\theta)$. The co-ordinates of this free-fall frame, with respect to our inertial frame would be $y=-g t^{2} / 2$. That is, $y=-g\left(x / v_{h}\right)^{2} / 2$.

Now translating back to the inertial frame the co-ordinates of the projectile becomes $y=x \tan (\theta)-g\left(x / v_{h}\right)^{2} / 2$ That is:

$$
y=-\frac{g \sec ^{2} \theta}{2 v_{0}^{2}} x^{2}+x \tan \theta
$$

(where $v_{0}$ is the original velocity, $\theta^{\prime}$ is the angle of distance from the ground, and $g$ is the acceleration due to gravity).

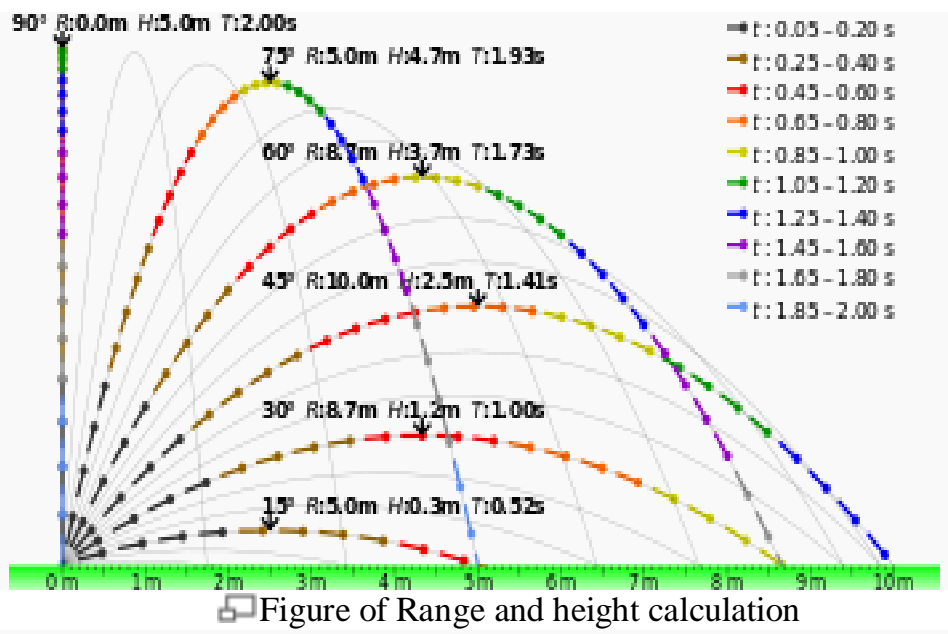

Trajectories of projectiles launched at special elevation angles but the same speed of $10 \mathrm{~m} / \mathrm{s}$ in a vacuum and standardized downward gravity field of $10 \mathrm{~m} / \mathrm{s}^{2}$. Points are at $0.05 \mathrm{~s}$ intervals and length of their tails is linearly proportional to their velocity. $t=$ time from begin, $T=$ time of air travel, $R=$ range and $H=$ highest point of trajectory (indicated with arrows).

The range, $R$, is the greatest distance the object schedule along the-axis in the I sector. The initial velocity, $v_{i}$, is the speed at which said object is launched from the spot of origin. The initial angle, $\theta_{i}$, is the angle at which said object is released. The $g$ is the respective gravitational pull on the object within a null-medium.

$$
R=\frac{v_{i}^{2} \sin 2 \theta_{i}}{g}
$$

The height, $h$, is the maximum parabolic height said object reaches within its trajectory

$$
h=\frac{v_{i}^{2} \sin ^{2} \theta_{i}}{2 g}
$$




\subsection{Zigbee Module:}

\section{Wireless Communication}

The XBee/XBee-PRO RF Modules are designed to function within the ZigBee protocol and support the unique necessities of inexpensive, low-power wireless antenna networks. The modules require minimal power and provide reliable delivery of data between remote devices. The modules activate within the ISM $2.4 \mathrm{GHz}$ frequency band and are compatible with the following:

- XBee RS-232 Adapter

- XBee RS-485 Adapter

- XBee Analog I/O Adapter

- XBee Digital I/O Adapter

- XBee Sensor Adapter

- XBee USB Adapter

- $\quad$ XStick

- Connect Port X Gateways

- XBee Wall Router.

The XBee/XBee-PRO ZB firmware can be installed on XBee modules. This firmware is well-suited with the ZigBee 2007 specification, while the ZNet 2.5 firmware is based on Ember's proprietary "planned for ZigBee" mesh stack (EmberZNet 2.5). ZB and ZNet 2.5 firmware are equal in environment, but not over-the-air compatible. Devices running ZNet 2.5 firmware cannot talk to devices running the ZB firmware.

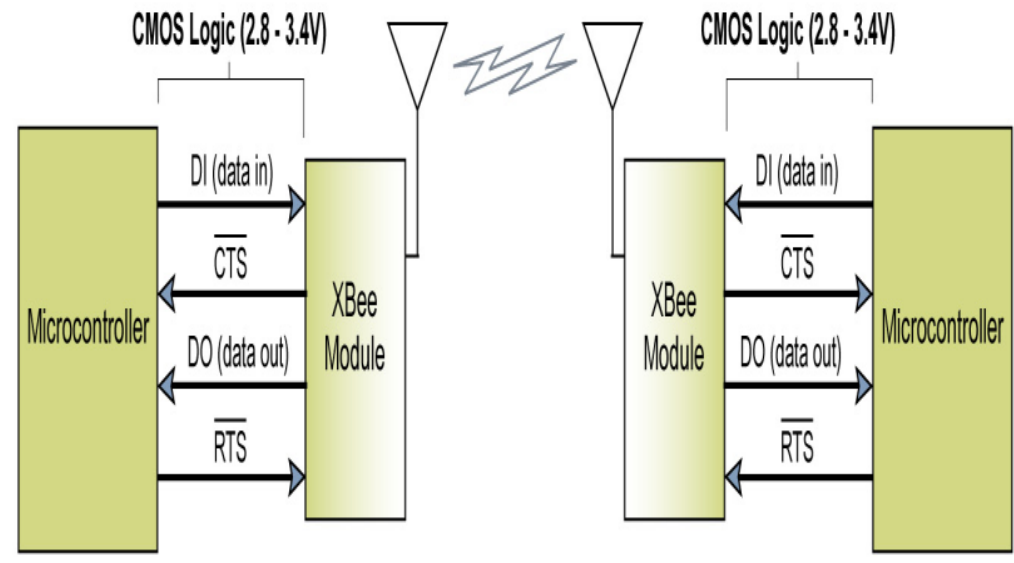

Fig5.Zigbee Module To Microcontroller

\section{Experimental Results}

In this part, the proposed of use of trajectory recognition algorithm is validated by the following two experiments.1)Hand written numeral recognition and 2)motion recognition. The proposed trajectory recognition algorithm consists of the subsequent actions: acceleration acquisition, signal preprocessing.

We collected the acceleration signals in a laboratory environment and displayed in the personal computer.

\section{Conclusion}

This paper presents an accelerometer-based digital pen for handwritten digit by with trajectory recognition applications.

The digital pen consists of a triaxial accelerometer, a microcontroller, and an Zigbee wireless transmission module for sensing and get-together the signals of accelerations of handwriting and gesture trajectories. By means of this technology we can put pen to paper \& display the characters not including the keyboard for applying the human interaction to the computer. 


\section{References}

[1] E. Sato, T. Yamaguchi, and F. Harashima, "Natural interface using pointing behavior for human-robot gestural interaction," IEEE Trans. Ind. Electron., vol. 54, no. 2, pp. 1105-1112, Apr. 2007.

[2] Y. S. Kim, B. S. Soh, and S.-G. Lee, “A new wearable input device: SCURRY,” IEEE Trans. Ind. Electron., vol. 52, no. 6, pp. 1490-1499, Dec. 2005.

[3] A. D. Cheok, Y. Qiu, K. Xu, and K. G. Kumar, "Combined wireless hardware and real-time computer vision interface for tangible mixed reality," IEEE Trans. Ind. Electron., vol. 54, no. 4, pp. 2174-2189, Aug. 2007.

[4] Z. Dong, U. C. Wejinya, and W. J. Li, "An optical-tracking calibration method for MEMS-based digital writing instrument," IEEE Sens. J., vol. 10, no. 10, pp. 1543-1551, Oct. 2010.

[5] J. S.Wang, Y. L. Hsu, and J. N. Liu, "An inertial-measurement-unit-based pen with a trajectory reconstruction algorithm and its applications,” IEEE Trans. Ind. Electron., vol. 57, no. 10, pp. 3508-3521, Oct. 2010.

[6] S.-H. P. Won, W. W. Melek, and F. Golnaraghi, "A Kalman/particle filter-based position and orientation estimation method using a position sensor/inertial measurement unit hybrid system," IEEE Trans. Ind. Electron., vol. 57, no. 5, pp. 1787-1798, May 2010.

[7] S.-H. P. Won, F. Golnaraghi, and W. W. Melek, "A fastening tool tracking system using an IMUand a position sensor with Kalman filters and a fuzzy expert system," IEEE Trans. Ind. Electron., vol. 56, no. 5, pp. 1782-1792, May 2009.

[8] Y. S. Suh, “Attitude estimation by multiple-mode Kalman filters," IEEE Trans. Ind. Electron., vol. 53, no. 4, pp. 1386-1389, Jun. 2006.

[9] J. Yang, W. Chang, W. C. Bang, E. S. Choi, K. H. Kang, S. J. Cho, and D. Y. Kim, "Analysis and compensation of errors in the input device based on inertial sensors," in Proc. IEEE Int. Conf. Inf. Technol.-Coding and Computing, 2004, pp. 790-796.

[10] Y. Luo, C. C. Tsang, G. Zhang, Z. Dong, G. Shi, S. Y. Kwok, W. J. Li, P. H. W. Leong, and M. Y. Wong, "An attitude compensation technique for a MEMS motion sensor based digital writing instrument," in Proc. IEEE Int. Conf. Nano/Micro Eng. Mol. Syst., 2006, pp. 909-914.

[11] Z. Dong, G. Zhang, Y. Luo, C. C. Tsang, G. Shi, S. Y. Kwok, W. J. Li, P. H. W. Leong, and M. Y. Wong, "A calibration method for MEMS inertial sensors based on optical tracking," in Proc. IEEE Int. Conf. Nano/Micro Eng. Mol. Syst., 2007, pp. 542-547.

[12] J. G. Lim, S. Y. Kim, and D. S. Kwon, "Pattern recognition-based realtime end point detection specialized for accelerometer signal," in Proc. IEEE/ASME Int. Conf. Adv. Intell. Mechatron., 2009, pp. 203-208.

[13] S. D. Choi, A. S. Lee, and S. Y. Lee, "On-line handwritten character recognition with 3D accelerometer," in Proc. IEEE Int. Conf. Inf. Acquisition, 2006, pp. 845-850.

[14] N. C. Krishnan, C. Juillard, D. Colbry, and S. Panchanathan, "Recognition of hand movements using wearable accelerometers," J. Ambient Intell. Smart Environ., vol. 1, no. 2, pp. 143-155, Apr. 2009.

[15] B. Milner, "Probabilistic neural networks," in Proc. Inst. Elect. Eng._Colloq. Doc. Image Process. Multimedia, 1999, pp. 5/1-5/6. 